\title{
Current concepts in the management of stable ischemic heart disease and acute coronary syndrome in patients with hemophilia
}

\author{
Ahmad Y. Jabbar, Hassan Baydoun, Maissaa Janbain, Keith C. Ferdinand \\ Department of Medicine, Tulane School of Medicine, Tulane Heart and Vascular Institute, New Orleans, LA, USA \\ Contributions: (I) Conception and design: AY Jabbar, H Baydoun; (II) Administrative support: KC Ferdinand; (III) Provision of study materials or \\ patients: All authors; (IV) Collection and assembly of data: All authors; (V) Data analysis and interpretation: All authors; (VI) Manuscript writing: All \\ authors; (VII) Final approval of manuscript: All authors. \\ Correspondence to: Ahmad Y. Jabbar, MD. Department of Medicine, Heart and Vascular Institute, 1430 Tulane Avenue SL-8548, New Orleans, \\ Louisiana 70112, USA. Email: ajabbar@tulane.edu.
}

\begin{abstract}
Coronary artery disease (CAD), including stable ischemic heart disease (SIHD) and acute coronary syndrome (ACS), remains the leading cause of death in the US and one of the primary modalities used in the treatment of CAD is percutaneous coronary intervention (PCI). Despite the potential benefits of PCI in high risk CAD patients, the risk of hemorrhage presents a dilemma in the treatment of patients with hemophilia A and B. In an attempt to provide guidance on the management of SIHD and ACS in patients with hemophilia, we present the case of a patient with moderate hemophilia B and ACS who subsequently underwent PCI followed by a review of the associated literature.
\end{abstract}

Keywords: Hemophilia; acute coronary syndrome (ACS); stable ischemic heart disease (SIHD)

Submitted Apr 22, 2018. Accepted for publication May 22, 2018.

doi: 10.21037/atm.2018.05.29

View this article at: http://dx.doi.org/10.21037/atm.2018.05.29

\section{Introduction}

Coronary artery disease (CAD), including stable ischemic heart disease (SIHD) and acute coronary syndrome (ACS), continues to be a public health concern, an economic burden, and remains the leading cause of death in the US. According to the American Heart Association (AHA) and American College of Cardiology (ACC), CAD accounts for 1 in 7 deaths in the US and the prevalence of myocardial infarction (MI) is about 7.9 million in US adults. The estimated direct and indirect costs of $\mathrm{MI}$ and CAD were $\$ 12.1$ billion and $\$ 9.0$ billion, respectively, and were two of the ten most expensive conditions treated in US hospitals (1). One of the primary modalities used in the treatment of CAD is percutaneous coronary intervention (PCI). Despite the potential benefits of PCI in high risk CAD patients, especially associated with ACS or severe symptoms, the risk of hemorrhage presents a dilemma, specifically in the treatment of certain populations, such as those with bleeding disorders.
In consideration of the above, patients with hemophilia $(\mathrm{PWH}) \mathrm{A}$ and $\mathrm{B}$ are at high risk for bleeding specifically after PCI. These conditions result from an absence or dysfunction of clotting factor VIII (FVIII) or IX (FIX). Moreover, these inherited X-linked recessive bleeding disorders occur almost exclusively in males (2). Severity of hemophilia is based on plasma concentrations of FVIII or FIX activity (severe $<1 \%$, moderate $1-5 \%$, and mild $5-40 \%$ normal activity) (2,3). In recent years hemophilia treatment centers (HTC) have improved the life expectancy of hemophiliacs, which has led to the development of age-related co-morbidities in this population, including atherosclerotic cardiovascular disease (ASCVD) (2). According to the Centers for Disease Control and Prevention (CDC), there were 8,816 new hemophilia patients between 2005-2009 and in 2016 the World Federation of Hemophilia reported data on 295,000 PWH in the world (4,5). The life expectancy for patients with severe hemophilia increased 5-fold from 11 to 56.8 years and from 27.5 to 71.5 years for moderate 
hemophilia between 1920 and 1980. Nevertheless, only $4 \%$ of those with severe hemophilia died from CAD during this period (6). However, the true prevalence of ASCVD is unclear. In an attempt to provide guidance on the management of SIHD and ACS in PWH, we present the case of a patient with moderate hemophilia B and ACS who subsequently underwent PCI followed by a review of the associated literature.

\section{Case report}

A 42-year-old male with moderate hemophilia B (residual FIX activity $3 \%$ ) was referred for coronary angiography because of typical angina and an abnormal treadmill exercise stress test with non-diagnostic ST segment depressions. His only ASCVD risk factor was hypertension and he required FIX infusions approximately once a year for occasional joint effusions and minor trauma. He had no major laboratory abnormalities and was able to receive $325 \mathrm{mg}$ of aspirin. Coronary angiography was performed, after consultation with a hematologist, using right radial artery access. Prior to the procedure, he received 6,800 international units (IU) $(80 \mathrm{IU} / \mathrm{kg})$ of recombinant FIX. Lidocaine was used for local anesthesia and insertion of a $5 \mathrm{Fr}$ arterial sheath was followed by an injection of $2.5 \mathrm{mg}$ of verapamil and $200 \mathrm{mg}$ of nitroglycerin into the sheath and 5,000 units of unfractionated heparin (UFH) systemically. A diagnostic angiogram of the left and right coronary systems was performed using JL 3.5 and JR 4 catheters respectively. A subtotal occlusion of $100 \%$ of the mid left anterior descending (LAD) artery was identified as the culprit lesion. At this time the $5 \mathrm{Fr}$ sheath was exchanged for a $6 \mathrm{Fr}$ sheath for intervention, and after multiple guides were unable to engage the left main ostium the procedure was converted to right groin access utilizing a $6 \mathrm{Fr}$ femoral artery sheath and a 6 Fr CLS 3.5 guide catheter. Additional UFH was given to achieve an activated clotting time (ACT) $>250 \mathrm{~s}$. The lesion was dilated with an apex $2.5 \mathrm{~mm} \times 12 \mathrm{~mm}$ balloon followed by the placement of a VeriFlex $3.5 \mathrm{~mm} \times 16 \mathrm{~mm}$ bare-metal stent (BMS). Immediately after the procedure, he was given an oral loading dose of $300 \mathrm{mg}$ of clopidogrel and hemostasis of the radial artery was achieved with the use of a TR Band, a radial artery compression device, and the femoral artery with manual pressure when the ACT was no longer therapeutic. Twenty-four hours after the procedure $6,800 \mathrm{IU}(80 \mathrm{IU} / \mathrm{kg})$ of FIX was administered and repeated again daily for a total of 3 days. The patient was discharged home with aspirin $81 \mathrm{mg}$ and clopidogrel $75 \mathrm{mg}$ daily after 3 days of observation with no bleeding complications. He continued to receive prophylaxis therapy with regular administration of FIX concentrates to keep trough levels around $15 \%$ while on dual antiplatelet therapy (DAPT). After 1 month, he was switched to aspirin alone and trough levels were maintained at $5 \%$.

\section{CAD and ASCVD risk in PWH}

In an effort to compare ASCVD comorbidities in the US, Pocoski et al. identified 2,506 PWH A and compared them to general matched cohort of 7,518 patients. In $\mathrm{PWH} \mathrm{A}$, proportions of CAD $(10.7 \%$ vs. $5.8 \%$, $\mathrm{P}<0.001)$, hypertension (22.6\% vs. $15.5 \%, \mathrm{P}<0.001)$, and hyperlipidemia $(15.9 \%$ vs. $11.9 \%, \mathrm{P}=0.001)$ were significantly greater (2). Biere-Rafi et al. examined 100 hemophilia A and B patients compared to 200 healthy subjects and found that the prevalence of risk factors such as hyperglycemia (24\%) and hypertension (51\%) was similar to the general population (7). Data collected from HTC and registries in the Netherlands and the United Kingdom demonstrated that in males with hemophilia over the age of 40 compared to the general population, the risk of ASCVD was greater using the QRISK 22011 score (8.9\% vs. $6.7 \%)(8)$. A study evaluating the cause of mortality of 6,018 hemophilia patients in the United Kingdom found that mortality from CAD was lower at only $62 \%$ of the general population rates (9). Berger et al. compared the data on ASCVD and risk factors in PWH aged $>40$ years with published German general population data and found $\mathrm{PWH}$ to have a higher prevalence of hypertension but a lower rate of CAD (10).

As a surrogate for evaluation of atherosclerosis, intima-media thickness (IMT) has been studied and has shown conflicting information in the evaluation of atherosclerosis for PWH. Sramek et al. compared 76 individuals with heritable bleeding disorders (hemophilia and von Willebrand disease) with 142 healthy controls and found no difference in IMT, whereas a case-control study comparing $50 \mathrm{PWH}$ with 50 age-matched controls revealed significantly lower IMT in patients with moderate or severe hemophilia $(11,12)$. Although it is often believed that severe hemophilia may offer protection against MI, hemophilia does not appear to reduce the incidence of ASCVD when patients have typical risk factors for ASCVD $(13,14)$. Overall, the rates of coronary atherosclerosis for $\mathrm{PWH}$ should be expected to 
be similar to the general population in consideration that $\mathrm{PWH}$ can have similar risk factors for ASCVD.

\section{Approaches to SIHD and ACS in PWH}

Caring for PWH in the setting of SIHD and ACS can be challenging, particularly if the hemophilia is severe. The management of ACS consists of treatment with aggressive antiplatelet and anticoagulant therapies, which poses a risk for PWH because of the obvious bleeding risks involved. To make matters more complicated, infusion of factor concentrates has been associated with the development of ACS (15). For such patients, hemophilia specialist consultation is typically necessary to help guide therapy for this complex disease (13). Additionally, patients with severe hemophilia appear less likely to undergo coronary artery bypass graft surgery (CABG) or PCI, perhaps due to increased complexity and concern for poor procedural outcomes. In fact, CABG is rarely done for patients with severe hemophilia while rates of PCI and CABG are at least comparable in patients with mild to moderate hemophilia (16).

Recently, Boehnel et al. conducted a systematic review to assess the periprocedural and long-term management of PWH undergoing PCI. It is the largest review to date, identified 54 patients, that attempts to summarize the management of PWH in the setting of ACS (17). Studies referenced in the ACC/AHA guidelines do not include PWH $(18,19)$. Case reports, small retrospective studies, and consensus statements have attempted to determine the best method to avoid bleeding in $\mathrm{PWH}$ while treating ACS, yet there are no clear guidelines available to aid clinicians $(13,16,20,21)$.

\section{Initial management}

Similar to the general population, beta blockers and nitrates are used in nearly all cases (16). PWH often receive replacement therapy with factor concentrates prior to any procedure. In addition, most patients are treated with prophylaxis on demand in case of bleeding episodes, whereas severe hemophiliacs require regular prophylaxis up to several times a week (16). The doses that have been reported set targets of FVIII and FIX levels ranged from $30 \%$ to $100 \%$. Periprocedural target peaks aim for about $80 \%$ and troughs aim for about $30 \%(16,17,22)$. Several cases (ranging from mild to severe disease) have been reported in which no hemostatic support was used during exposure to aspirin with or without heparin, and no excessive bleeding was reported $(15,16)$.

Fogarty et al. noted that 15 out of 20 cases did not alter the initial management of ACS in PWH and when it was modified it usually was withholding or delaying aspirin or lowering the dose of low molecular weight heparin (LMWH) (16). Hemostatic support before and following antithrombotic therapy included FVIII or FIX boluses or boluses followed by infusions with troughs ranging from $\geq 30 \%$ to $\geq 50 \%$, and/or FVIII or FIX peaks ranging from $\geq 70 \%$ to $\geq 100 \%(16,17,22)$. A more general recommendation prior to PCI is to keep peak levels of clotting factor infused at concentrate above $80 \%$ and continued for 48 hours after the procedure (14). The assessment by the age-related developments and comorbidities in hemophilia (ADVANCE) group favored the administration of replacement therapy prior to PCI, ideally achieving a peak clotting factor level exceeding $80 \%$ (13). In patients with baseline clotting factor $\geq 25 \%$, there may not be a need to give prophylaxis prior to planned PCI or coronary angiogram (23). Desmopressin has been traditionally used to correct clotting factor deficiency in PWH. Because of the risk of increase in heart rate, higher diastolic pressures, and risk of arterial thrombosis, desmopressin is not recommended in patients with ischemic heart disease (20).

\section{Choice of arterial access site}

Most cases reported in the literature used femoral access (up to $52 \%$ ), including our case, with rare major bleeding events (17). Radial access reduces the risk of bleeding, including major bleeding, and therefore should always be considered as the primary access site especially for highrisk patients such as $\mathrm{PWH}$ presenting with ACS. Previous recommendations have been to avoid femoral arterial access in PWH given the increased risk of bleeding compared to radial access (13). Despite this recommendation, there has been a variation in several studies in the selection of arterial access sites and the outcomes of bleeding. Fogarty et al. reported in seven patients undergoing PCI, and all but one had femoral access site used for the procedure (16). On the other hand, Fefer et al. reported a case series of three patients with ACS, all used radial access (15). Therefore, given that radial access is associated with a reduction in mortality in ST-elevation MI (STEMI) patients, reduced risk of access site bleeding, and ease of control of bleeding, it has been proposed that radial access is the ideal choice in 
PWH $(14,19)$. Although this has been the recommendation, many of the cases reported used femoral access with few complications or bleeding from the access site. Schutgens et al. recommended radial access in their institutional guidelines based on the fact that up to $70 \%$ of all major bleeding complications (access site hematomas and retroperitoneal bleeds) were due to femoral access (20).

\section{Choice of stent}

Although patients with drug-eluting stents (DES) have a lower risk of repeat revascularization compared to BMS, they require longer treatment with antiplatelet agents $(18,19)$. A recent study evaluating DES vs. BMS in the setting of stable and unstable CAD found no difference in the composite outcome of death or MI concluding that BMS is still a viable option in such patients (24). In the study conducted by Fogarty et al., six patients received BMS and 1 received DES (16). Fefer et al. reported that all three cases received BMS, one patient had in-stent restenosis one year later and underwent cutting balloon angioplasty with no further complications (15). The use of BMS with reduced thrombogenic risk such as titanium-nitric oxide coated stents, carbofilm coated stents, or endothelial progenitor cell capture stents are preferred, if available (15). In patients with residual clotting factor level of $25 \%$ or higher, DES is considered a viable option (23). With the advances in DES and the need for less time on antiplatelet agents, this recommendation may be expanding. Similar to the general population, the risk of restenosis is still present (25). Patients have been shown to have $50-70 \%$ restenosis as soon as 6-12 months post PCI while off DAPT (26). This has not been evaluated in patients with bleeding disorders and there is no evidence to suggest that restenosis rates are similar to the general population.

\section{Choice of antithrombotic agent}

UFH has a very short half-life making it favorable to LMWH for PCI in hemophilia. Moreover, UFH anticoagulation effect can be easily measured in the lab by the ACT and reversed by protamine sulfate. According to Fogarty et al., UFH was used in all seven patients that underwent PCI. Hemostatic support with FXIII and FIX concentrates during PCI or CABG included bolus infusion, continued infusion, or both. None of the PCI patients received blood components within 24 hours after PCI and all achieved successful revascularization (16). In the cases reported by Fefer et al., choice of antithrombotic agents included standard weight-adjusted bolus and continuous infusion of bivalirudin in one patient and UFH in the two other patients (15). Several case studies have shown that bivalirudin can be used safely with no complications during PCI in hemophilia patients (14).

\section{Choice of antiplatelet agent}

DAPT is recommended after every PCI to prevent stent thrombosis $(18,19)$. In most of the cases reported, aspirin along with clopidogrel was used for long-term DAPT. A case of unstable angina and LAD in-stent restenosis was reported, in order to avoid DES placement cutting balloon dilatation was used with a 1 -year follow-up thallium scan that showed no anterior wall ischemia (15). There are currently no recommendations about the choice of antiplatelet agents, but one must keep in mind the properties of each agent and risks involved in using each. Clopidogrel and prasugrel are prodrugs and are irreversible, whereas ticagrelor is direct acting and reversible. Recent studies have shown that ticagrelor and prasugrel are superior to clopidogrel in reducing ischemic events, with the risk of higher bleeding $(14,19)$. The AHA/ACC guidelines recently made changes that allowed the use of ticagrelor and prasugrel early on in the treatment of ACS, given the bleeding risk it is difficult to extrapolate this to $\mathrm{PWH}$ population (19). PWH should always be taking a proton pump inhibitor along with DAPT in order to reduce the risk of gastrointestinal bleeding. In addition, Cayla et al. recommend that glycoprotein IIb/IIIa inhibitors should be limited to patients with a highburden thrombus during coronary angiography or in cases of slow or no-reflow or thrombotic complications (14). Also, daily aspirin without prophylaxis has been recommended as a reasonable approach by Schutgens et al., especially in patients with severe disease given the much higher risk of bleeding. When bleeding frequency increases, aspirin should be stopped (20). In the prospective study, Tuinenburg et al. recommended extension of DAPT therapy without clotting factors in patients with residual clotting factor of $25 \%$ or higher (23).

\section{Duration of antiplatelet agents}

The duration of antithrombotic agents should be as short as possible with an easy reversal given the risk of periprocedural 
and long-term bleeding risks. In the setting of an elective PCI for SIHD, BMS can be stopped after 1 month and DES after 6 months per the current guidelines; recent studies have also suggested less than 6 months with new generation DES (23). Therefore, BMS should be recommended to facilitate a shorter duration of DAPT. Fogarty et al. reported that all patients received aspirin and clopidogrel for 1-12 months followed by aspirin indefinitely and clotting factor prophylaxis during this time was used in almost all patients with severe hemophilia A (16). In PWH if BMS is used it is recommended that 4 weeks of DAPT be given, otherwise 6-12 months has been safe in the setting of DES use.

\section{Management of bleeding risk associated with anticoagulation and antiplatelet therapy}

According to the ADVANCE working group recommendations, a trough level of $5-15 \%$ should be maintained while on DAPT after PCI (13). Only 2 of the 17 patients reported by Fogarty et al. experienced bleeding while receiving antiplatelet agents. One patient had mild hemophilia A and STEMI and received no secondary prophylaxis, DAPT for 6 months, and experienced severe gastrointestinal bleeding. One patient, with severe hemophilia A and non-STEMI (NSTEMI), was treated medically but required an increase in FVIII replacement after 2 months of single-agent aspirin because of nose bleeding and excessive bruising (16). In addition, Fefer et al. reported that all three patients had no significant bleeding on follow-up (15). There have also been reports of patients that did not receive any FVIII replacement therapy in the setting of ACS and receiving heparin and antithrombotics without any bleeding events (27). Schutgens et al. originally recommended aiming for a clotting factor trough level of $30 \%$ by inducing clotting factor concentrate every 12 hours during DAPT. In the prospective follow-up the group felt that daily dosing was adequate in patients without severe disease (20). A recent case report by Chang et al. discusses a patient with multivessel PCI and history of bleeding being treated with recombinant FVIII and trough level above 15 during DAPT. This patient was noted to have several episodes of bleeding at this trough level and during a subsequent PCI, he was maintained at a trough level $>20$ with no bleeding episodes (26). There have been several recommendations in regards to trough goals for replacement therapy, we believe that aiming for a trough clotting factor level of $\geq 30 \%$ is ideal and leads to the least amount of bleeding

\section{Management of patients undergoing (CABG)}

Fogarty et al. reported that all six patients who underwent CABG had experienced unstable angina or NSTEMI, and all had cardiac catheterization findings that were not amenable to PCI. As perioperative hemostatic support, one patient received FIX by continuous infusion targeting a trough level above $100 \%$. Three patients received a single bolus of FVIII concentrates followed by continuous infusion. A patient with mild hemophilia A received desmopressin perioperatively. Another patient with mild hemophilia A and a history of an inhibitor and poor FVIII recovery received recombinant activated FVII. Adjunctive antifibrinolytic drugs were used in three cases with mild/moderate hemophilia A. Two patients received blood components [e.g., packed red blood cells (pRBC)] administered routinely postsurgically in one case and pRBCs plus platelets plus fresh frozen plasma in another case with established chronic hepatitis $\mathrm{C}$ virus (HCV) infection (16).

\section{Conclusions}

Staritz et al. reviewed the European Society of Cardiology Guidelines and used the Delphi-like methodology to develop expert consensus on the key aspect of clinical management of PWH presenting with ACS (13). Given the data that is available from various case reports and hospital-based guidelines, we consider a similar approach to treating SIHD and ACS in PWH as summarized in Table 1. Initial management should not differ in $\mathrm{PWH}$ other than considering prophylactic treatment with replacement factor based on severity, ideally in all PWH. Radial artery should be the preferred access site. UFH has a short half-life and presents a lower risk of bleeding, therefore it is preferred over LMWH. Clopidogrel has been shown to have a lower risk of bleeding compared to ticagrelor and prasugrel and therefore should be used in PWH. The duration of DAPT should be as short as possible given the higher risk of bleeding and depending on the type of stent (BMS $v s$. DES) and the clinical presentation (elective PCI $v s$. ACS). In regards to surgical management of ACS in PWH, consider it on a case by case scenario. At all times consider consulting a hematologist on expert opinion to assist in the management of these patients. 
Table 1 Recommendations for management of acute coronary syndrome in patients with hemophilia

\begin{tabular}{ll}
\hline Management & Recommendations \\
\hline Initial management & $\begin{array}{l}\text { Clotting factor correction aiming for a peak level of } 80 \% \text { for } 48 \text { hours with a trough level of } 30 \% \text { after } 48 \\
\text { hours and as long as DAPT is given }\end{array}$ \\
$\begin{array}{l}\text { Arterial access site } \\
\text { Choice of stent }\end{array}$ & BMS if possible. May change soon given advances in DES \\
Antithrombotic therapy & UFH (bolus infusion $70 \mathrm{U} / \mathrm{kg}$ followed by continues infusion of $400 \mathrm{U} / \mathrm{kg} /$ day for 48 hours). May consider \\
Antiplatelet therapy & Oral loading dose of $600 \mathrm{mg}$ clopidogrel before percutaneous coronary intervention, followed by 75 mg \\
& clopidogrel daily for 4 weeks with trough clotting factor $\geq 30 \%$
\end{tabular}

DAPT, dual antiplatelet therapy; BMS, bare-metal stent; DES, drug-eluting stent; UFH, unfractionated heparin; LMWH, low molecular weight heparin.

\section{Acknowledgements}

None.

\section{Footnote}

Conflicts of Interest: Dr. KC Ferdinand is a consultant for Amgen, Sanofi, Boehringer-Ingelheim, Quantum Genomics, and Novatis. The other authors have no conflicts of interest to declare.

\section{References}

1. Benjamin EJ, Virani SS, Callaway CW, et al. Heart Disease and Stroke Statistics-2018 Update: A Report From the American Heart Association. Circulation 2018;137:e67-492.

2. Pocoski J, Ma A, Kessler CM, et al. Cardiovascular comorbidities are increased in U.S. patients with haemophilia A: a retrospective database analysis. Haemophilia 2014;20:472-8.

3. Mannucci PM, Schutgens RE, Santagostino E, et al. How I treat age-related morbidities in elderly persons with hemophilia. Blood 2009;114:5256-63.

4. Centers for Disease Control and Prevention. Report on the Universal Data Collection Program. The Universal Data Collection Program 2005;7:1-39.

5. World Federation of Hemophilia. Report of the Annual Global Survey 2016. 2016;October:3-73. Available online: http://www1.wfh.org/publications/files/pdf-1690.pdf

6. de Raucourt E, Roussel-Robert V, Zetterberg E. Prevention and treatment of atherosclerosis in haemophilia - how to balance risk of bleeding with risk of ischaemic events. Eur J Haematol 2015;94 Suppl 77:23-9.

7. Biere-Rafi S, Baarslag MA, Peters M, et al. Cardiovascular risk assessment in haemophilia patients. Thromb Haemost 2011;105:274-8.

8. Fransen van de Putte DE, Fischer K, Makris M, et al. Unfavourable cardiovascular disease risk profiles in a cohort of Dutch and British haemophilia patients. Thromb Haemost 2013;109:16-23.

9. Darby SC, Kan SW, Spooner RJ, et al. Mortality rates, life expectancy, and causes of death in people with hemophilia A or B in the United Kingdom who were not infected with HIV. Blood 2007;110:815-25.

10. Berger K, Schopohl D, Lowe G, et al. How to compare cardiovascular disease and risk factors in elderly patients with haemophilia with the general population. Haemophilia 2016;22:e406-16.

11. Sramek A, Reiber JH, Gerrits WB, et al. Decreased coagulability has no clinically relevant effect on atherogenesis: observations in individuals with a hereditary bleeding tendency. Circulation 2001;104:762-7.

12. Bilora F, Zanon E, Petrobelli F, et al. Does hemophilia protect against atherosclerosis? A case-control study. Clin Appl Thromb Hemost 2006;12:193-8.

13. Staritz P, de Moerloose P, Schutgens R, et al. Applicability of the European Society of Cardiology guidelines on management of acute coronary syndromes to people with haemophilia - an assessment by the ADVANCE Working Group. Haemophilia 2013;19:833-40.

14. Cayla G, Morange PE, Chambost H, et al. Management of cardiovascular disease in haemophilia. Thromb Res 2013;132:8-14.

15. Fefer P, Gannot S, Lubetsky A, et al. Percutaneous coronary intervention in patients with haemophilia 
presenting with acute coronary syndrome: an interventional dilemma: case series, review of the literature, and tips for management. J Thromb Thrombolysis 2013;35:271-8.

16. Fogarty PF, Mancuso ME, Kasthuri R, et al. Presentation and management of acute coronary syndromes among adult persons with haemophilia: results of an international, retrospective, 10-year survey. Haemophilia 2015;21:589-97.

17. Boehnel C, Rickli H, Graf L, et al. Coronary angiography with or without percutaneous coronary intervention in patients with hemophilia-Systematic review. Catheter Cardiovasc Interv 2017. [Epub ahead of print].

18. Amsterdam EA, Wenger NK, Brindis RG, et al. 2014 AHA/ACC Guideline for the Management of Patients with Non-ST-Elevation Acute Coronary Syndromes: a report of the American College of Cardiology/American Heart Association Task Force on Practice Guidelines. J Am Coll Cardiol 2014;64:e139-228.

19. Levine GN, Bates ER, Blankenship JC, et al. 2015 ACC/ AHA/SCAI Focused Update on Primary Percutaneous Coronary Intervention for Patients With ST-Elevation Myocardial Infarction: An Update of the 2011 ACCF/ AHA/SCAI Guideline for Percutaneous Coronary Intervention and the 2013 ACCF/AHA Guideline for the Management of ST-Elevation Myocardial Infarction. J Am Coll Cardiol 2016;67:1235-50.

20. Schutgens RE, Tuinenburg A, Roosendaal G, et al. Treatment of ischaemic heart disease in haemophilia

Cite this article as: Jabbar AY, Baydoun H, Janbain M, Ferdinand KC. Current concepts in the management of stable ischemic heart disease and acute coronary syndrome in patients with hemophilia. Ann Transl Med 2018;6(15):299. doi: 10.21037/ atm.2018.05.29 patients: an institutional guideline. Haemophilia 2009;15:952-8.

21. Mannucci PM. Management of antithrombotic therapy for acute coronary syndromes and atrial fibrillation in patients with hemophilia. Expert Opin Pharmacother 2012;13:505-10.

22. Reilley MJ, Blair A, Matthai WH, et al. Revascularization strategies and in-hospital management in acute coronary syndromes complicated by hemophilia A or hemophilia B. Blood Coagul Fibrinolysis 2017;28:650-7.

23. Tuinenburg A, Damen SA, Ypma PF, et al. Cardiac catheterization and intervention in haemophilia patients: prospective evaluation of the 2009 institutional guideline. Haemophilia 2013;19:370-7.

24. Bonaa KH, Mannsverk J, Wiseth R, et al. Drug-Eluting or Bare-Metal Stents for Coronary Artery Disease. N Engl J Med 2016;375:1242-52.

25. Anderson JL, Morrow DA. Acute Myocardial Infarction. N Engl J Med 2017;376:2053-64.

26. Chang CY, Lin SY, Tsai JR, et al. Successful multivessel percutaneous coronary intervention for acute coronary syndrome in a HIV-positive severe haemophiliac. Haemophilia 2016;22:e481-4.

27. Zimmermann R, Staritz P, Huth-Kuhne A. Challenges in treating elderly patients with haemophilia: a focus on cardiology. Thromb Res 2014;134 Suppl 1:S48-52. 